University of Nebraska - Lincoln

DigitalCommons@University of Nebraska - Lincoln

$7-2009$

\title{
MFM studies of interlayer exchange coupling in Co/Ru/Co films: Effect of Ru layer thickness
}

\author{
Lanping Yue \\ University of Nebraska-Lincoln, lyue2@unl.edu \\ Zhen Li \\ University of Nebraska-Lincoln \\ Roger D. Kirby \\ University of Nebraska-Lincoln, rkirby1@unl.edu \\ David J. Sellmyer \\ University of Nebraska-Lincoln, dsellmyer@unl.edu
}

Follow this and additional works at: https://digitalcommons.unl.edu/physicssellmyer

Part of the Physics Commons

Yue, Lanping; Li, Zhen; Kirby, Roger D.; and Sellmyer, David J., "MFM studies of interlayer exchange coupling in Co/Ru/Co films: Effect of Ru layer thickness" (2009). David Sellmyer Publications. 220. https://digitalcommons.unl.edu/physicssellmyer/220

This Article is brought to you for free and open access by the Research Papers in Physics and Astronomy at DigitalCommons@University of Nebraska - Lincoln. It has been accepted for inclusion in David Sellmyer Publications by an authorized administrator of DigitalCommons@University of Nebraska - Lincoln. 


\title{
MFM studies of interlayer exchange coupling in $\mathrm{Co} / \mathrm{Ru} / \mathrm{Co}$ films: Effect of Ru layer thickness
}

\author{
Lanping Yue, ${ }^{1}$ Zhen Li, ${ }^{1,2}$ Roger Kirby, ${ }^{1,2}$ and David Sellmyer ${ }^{1,2}$ \\ ${ }^{1}$ Nebraska Center for Materials and Nanoscience, University of Nebraska-Lincoln, Lincoln, NE 68588, USA \\ ${ }^{2}$ Department of Physics and Astronomy, University of Nebraska-Lincoln, Lincoln, NE 68588, USA \\ Corresponding author - L. Yue, tel 402 472-2742, fax 402 472-2879, email lyue2@unl.edu
}

\begin{abstract}
Antiferromagnetically coupled magnetic thin films are promising candidates for the design of new magnetic storage and logic devices. The ability to control the interlayer thickness, therefore the magnetic reversal response, of exchange-coupled magnetic layers is of paramount importance in nanotechnology, especially in magnetic sensing element design and applications. In this work, magnetic force microscopy (MFM) with improved sensitivity and high spatial resolution probes was used to obtain a more detailed view of magnetization reversal behavior and domain evolution in the indirect exchange-coupled trilayer system: Co/Ru/ Co. The effect of the variable Ru interlayer thickness on the exchange coupling and thus the magnetic domain structure during the ferromagnetic (FM)/antiferromagnetic (AF) coupling transition in Co/Ru/Co films is well demonstrated. The MFM images display a distinct signature of AF coupling for the films with Ru thickness of $0.4 \mathrm{~nm}$. MFM has proven to be an effective tool for detecting FM/ AF interlayer coupling and exploring magnetic domain structures in exchange-coupled layered thin films.
\end{abstract}

Keywords: MFM, micromagnetic structures, exchange-coupled ultrathin films, high-resolution MFM probe, antiferromagnetically coupled domains

\section{Introduction}

Interlayer exchange-coupled ferromagnetic (FM)/antiferromagnetic (AF) layered thin films are essential components for the design of magnetic storage and logic devices, such as spin valves, magnetic recording media, memory devices, and magnetoresistive sensors $[1,2]$. They are expected to have many advantages over single layers, including improved density and stability, enhanced switching speed, and higher reliability of the switching process [3-5]. Since the first observation of an antiferromagnetic coupling between ferromagnetic layers through a nonmagnetic spacer [6], interlayer exchange coupling has been the subject of numerous studies [7-18]. It has been discovered that the coupling can be ferromagnetic or antiferromagnetic depending on the spacer layer thickness $[7,15]$. In this work, we focus on the study of interlayer thickness effects on magnetic domain structure during the FM-AF coupling transition by varying the thickness of the Ru spacer in interlayer exchange-coupled $\mathrm{Co} / \mathrm{Ru} / \mathrm{Co}$ thin films with inplane magnetic anisotropy. We demonstrate here the evidence of AF coupling between FM layers, which provide a convenient model system for studying the physics of antiferromagnetic films and surfaces. New magnetic materials with interesting physical properties might evolve from this work. Since the application of small magnetic elements requires a detailed understanding of their magnetic nanostructures and the mag- netization dynamics, the observed microscopic domain structures, magnetic transition behavior, and dynamical properties are of great interest to investigate. In particular, magnetic force microscopy (MFM) is a useful and sensitive technique to characterize local magnetic images by using improved high-resolution (about $15 \mathrm{~nm}$ ) MFM probes [19].

\section{Experimental methods}

A series of trilayer thin films with the synthetic structure consisting of two Co layers separated by a nonmagnetic Ru layer was deposited on silicon substrates by magnetron sputtering. For our sputtering setup, the Co thickness was fixed at $5 \mathrm{~nm}$ on both sides of the Ru layer, and the Ru spacer thickness $(x)$ was varied from 0 to $1 \mathrm{~nm}$ with a step of $0.1 \mathrm{~nm}$.

MFM was used to obtain a more detailed view of magnetization reversal behavior and domain configurations in as-deposited $\mathrm{Co}(5 \mathrm{~nm}) / \mathrm{Ru}(x) / \mathrm{Co}(5 \mathrm{~nm})$ thin films. MFM imaging was performed in the tapping/lift mode with a lift height of $15 \mathrm{~nm}$, operated at room temperature in air using a DI Dimension 3100 SPM. The high spatial resolution MFM probes [19] were made by coating a thin magnetic film on a cantilever with high coercivity and low moment. The tips were magnetized perpendicular to the sample surface, pointing downward. It is emphasized that the high coercivity and low moment MFM tip modified by focused-ion-beam milling not only improves the resolution of 
the MFM domain images, but also reduces the magnetic stray field of the tip, so that the local information on magnetic nanostructures of the soft magnetic samples will not be influenced by the very small stray magnetic field of the tip. The average magnetic properties of samples in magnetic fields up to $12 \mathrm{kOe}$ were determined using a MicroMag alternating gradient magnetometer (AGM). Magnetization precession was measured by a femtosecond-laser-based pump-probe system with direct optical excitation by varying the delay time between intense laser pulse (pump) and weak laser pulse (probe) [20].

\section{Results and discussion}

Figure 1 displays the evolution of MFM images of the magnetic domain and domain-wall structures of as-deposited Co/ $\mathrm{Ru} / \mathrm{Co}$ trilayers with increasing $\mathrm{Ru}$ interlayer thickness $(x)$ from 0 to $1 \mathrm{~nm}$. The scan size for all of images is $8 \times 8 \mu \mathrm{m}^{2}$.

When the Ru spacer $x=0$, Co thin film has a general ferromagnetic coarse-grained stripe domain structure. The bright and dark contrast of domains corresponds to high concentrations of positive and negative poles. For $x=0.2 \mathrm{~nm}$, the image shows a complicated ripple domain pattern. For $x=0.3 \mathrm{~nm}$, the bright and dark domain walls are observed as mesh-type ripples. For $x=0.4 \mathrm{~nm}$, the closed-loop domain walls appear as dominantly black bands between antiparallel "yellow" domains. In other words, large AF domains separated by high-contrast dark domain walls. Increasing the thickness of Ru interlayer $(x \geq 0.5 \mathrm{~nm})$ will decrease the AF coupling strength between the two magnetic layers in general, which results in the formation of complicated stripe domain structures. This series of MFM images of the $\mathrm{Co} / \mathrm{Ru} / \mathrm{Co}$ trilayer as a function of $x$ shows clearly that the exchange-coupling strength and thus the domain configuration are very sensitive to the thickness of the Ru interlayer.

Depending on the thickness of $\mathrm{Ru}$, the $\mathrm{Co} / \mathrm{Ru} / \mathrm{Co}$ thin films have two types of interlayer exchange coupling: FM and AF coupling. For FM domains, the magnetization changes from one stripe domain to the next, corresponding to the areas with the contrast of light and dark, respectively. For AF domains and domain-wall structures, the antiparallel arrays of magnetization in two Co layers occupy most of the area in the image form the contrast of yellow domains, while the parallel channels at the boundary of the antiparallel arrays form dark contrast closed-loop domain walls, suggestive of antiferromagnetic interlayer coupling between the top and bottom Co layers $[15,21]$. Clearly, the interlayer thickness is a large factor during the FM-AF transition in $\mathrm{Co} / \mathrm{Ru} / \mathrm{Co}$ films. For Ru thickness $x$ less than $0.2 \mathrm{~nm}$, the trilayer appears to be FM coupled; when $x \sim 0.3 \mathrm{~nm}$, the AF coupling through $\mathrm{Ru}$ becomes comparable to the FM coupling; at $x=0.4 \mathrm{~nm}$, AF coupling across the Ru interlayer is observed. The variation of domains and domain-wall formation with Ru thickness provides insight into the FM-AF transition behavior, which enable us to see how the relative orientation of Co magnetization vectors in a FM/AF/FM structures varies gradually from parallel to antiparallel orientation as a function of $\mathrm{Ru}$ thickness varying from 0 to $0.4 \mathrm{~nm}$.

The very different MFM domain structures between FM and AF-coupled thin films were also observed in Co/Ru superlattices with perpendicular anisotropy [22]. Although we cannot directly compare the domain structures between the AF and FM coupled samples for these two different kinds of thin film systems, both the earlier results [22] and the present work clearly indicate that MFM offers a reliable method for visualization of magnetic domains with high spatial resolution and sensitivity in exchange-coupled layered thin films.

The FM-AF transition behavior of $\mathrm{Co} / \mathrm{Ru} / \mathrm{Co}$ films described above was confirmed by AGM techniques, which measures the magnetic moments averaged over the entire thickness of the film. Figure 2 shows the in-plane magnetic hysteresis loops at room temperature for three $\mathrm{Co} / \mathrm{Ru} / \mathrm{Co}$ samples with the $\mathrm{Ru}$ spacer of $0.2,0.3,0.4 \mathrm{~nm}$, respectively. The arrows in the figures show the different states of magnetization for Co layers. The decreasing squareness of the loop with the increasing $\mathrm{Ru}$ thickness $x$ indicates that the ratio of FM to AF contributions decreased. The results are in good agreement with the MFM domain configurations, which confirms that the exchange coupling between Co layers is FM for $x=0.2 \mathrm{~nm}$ and AF for $x=0.4 \mathrm{~nm}$. When adjacent Co layers are AF coupled with the Ru spacer at appropriate thickness of $0.4 \mathrm{~nm}$, a small magnetic moment is observed in the hysteresis loop at the remanent state after saturation in an in-plane $12 \mathrm{kOe}$ field. The observed remanence may be due to some regions of FM coupling, or due to a biquadratic AF coupling [23].

Figure 3 shows the pump-probe measurement of ultrafast magnetization dynamics in interlayer exchange-coupled Co/
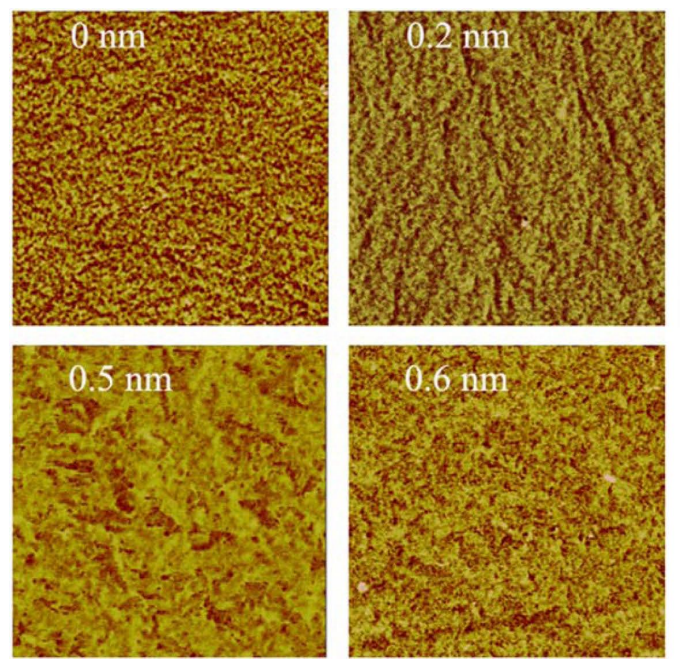
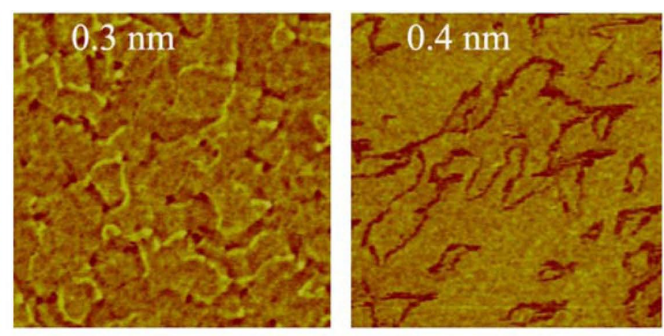
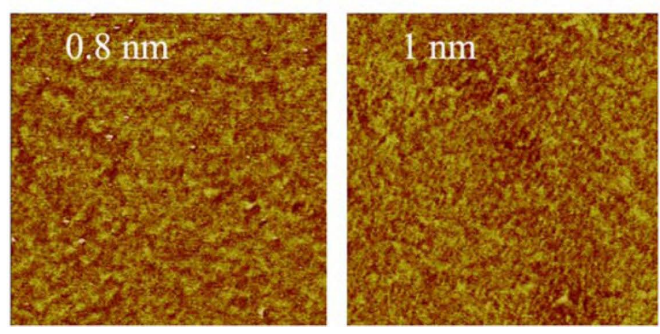

Figure 1. The evolution of MFM images of the magnetic domain and domain-wall structures of as-deposited $\mathrm{Co}(5 \mathrm{~nm}) / \mathrm{Ru}(x) / \mathrm{Co}(5 \mathrm{~nm})$ thin films with increasing Ru thickness gradually from 0 to $1 \mathrm{~nm}$. Each image is $8 \times 8 \mu \mathrm{m}^{2}$ in size. 

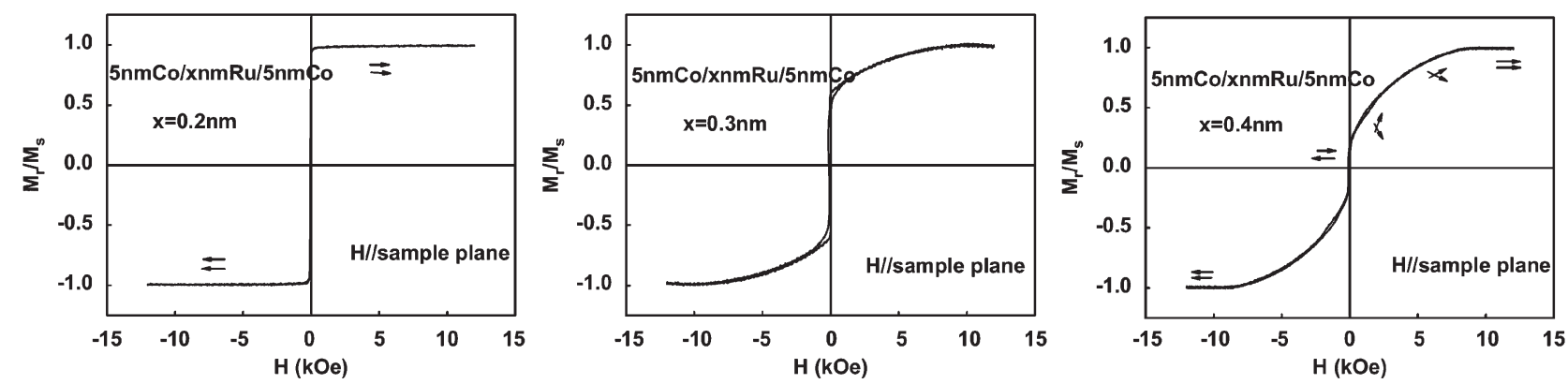

Figure 2. Magnetic hysteresis loops for $\operatorname{Co}(5 \mathrm{~nm}) / \operatorname{Ru}(x) / \operatorname{Co}(5 \mathrm{~nm})$ trilayer samples with $x=0.2,0.3,0.4 \mathrm{~nm}$, respectively. The arrows in the figures show the different states of magnetization for Co layers.

a

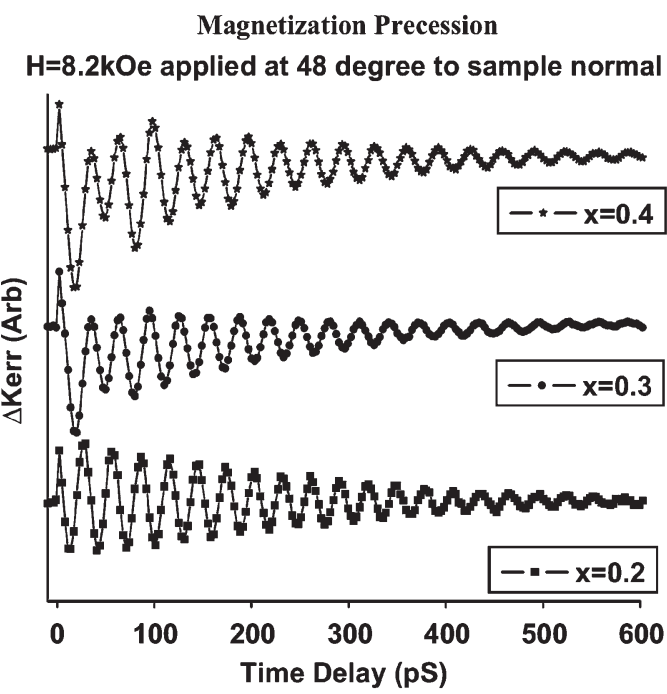

b

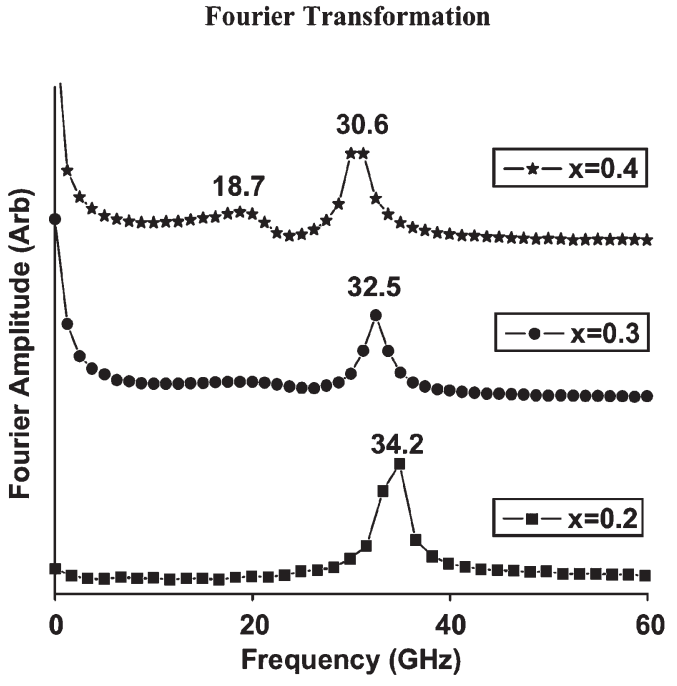

Figure 3. Pump-probe measurement of ultrafast magnetization dynamics in interlayer exchange-coupled Co/ Ru/Co thin films with various spacer thicknesses $(x=0.2,0.3,0.4 \mathrm{~nm}$ ): (a) $\Delta$ Kerr signal as a function of delay time and (b) the discrete Fourier transform (DFT) frequency spectrum of the corresponding data in (a).

$\mathrm{Ru} /$ Co thin films with various spacer thicknesses $(x=0.2,0.3$, $0.4 \mathrm{~nm})$. The $\Delta$ Kerr signal as a function of delay time is displayed in Figure 3(a); Figure 3(b) shows the discrete Fourier transform (DFT) frequency spectrum of the corresponding data in (a). It is found that for the AF-coupled sample $(x=$ $0.4 \mathrm{~nm})$, two precession frequencies are observed in the DFT, which can be identified as optical (low $f$ ) and acoustic (high $f$ ) precession modes [24]. For FM coupled sample $(x=0.2 \mathrm{~nm})$, only one acoustic mode is observed.

It is known that the exchange-coupling parameters depend on the nanostructural properties of the specimen, such as the pinhole density in the spacer, interface roughness, and also the magnetic domain structure and morphology of the layers $[18,25,26]$. Its strength can be controlled simply by varying the interlayer spacer thickness as demonstrated in our work here. Further work into the determination of the values of exchange-coupling constants from the frequencies of the optical and/or acoustical mode with accounting for the magnetic field strength and angle dependence will be accomplished by a twolayer Landau-Lifshitz-Gilbert-based model [20].

\section{Conclusion}

The effect of the variable Ru spacer thickness on the exchange coupling of $\mathrm{Co} / \mathrm{Ru} / \mathrm{Co}$ films is well demonstrated by com- bined studies involving MFM, AGM magnetometry, and dynamic methods. MFM images of both AF and FM domain structures revealed a clear domain-wall transition between the two types of magnetic orders upon changing the interlayer thickness. AF-coupled domains with high-contrast irregular closed-loop domain walls are observed for the films with $\mathrm{Ru}$ thickness of $0.4 \mathrm{~nm}$. The magnetic hysteresis loops also show a clear evidence of a FM-AF coupling transition as the Ru thickness is varied from 0.2 to $0.4 \mathrm{~nm}$. The dynamics measurements revealed two precession modes (optical and acoustic) in the AF-coupled samples. The experimental results show that the magnetic nanostructure and properties of samples are closely related to the thickness of the Ru interlayer. The interlayer exchange-coupled trilayer $\mathrm{Co} / \mathrm{Ru} / \mathrm{Co}$ films could be a good model system for studying the effect of antiferromagnetic (or ferromagnetic) exchange coupling on the domain formation and stabilization. Such coupled systems are of strong interest due to their technological relevance, which offer a new route to improved design of advanced magnetic devices.

\section{Acknowledgments}

We would like to thank Steven Michalski for help with magnetization precession measurements. This research is supported by the National Science Foundation through the Materials Re- 
search Science and Engineering Center (MRSEC) under Grant NSF-DMR-0820521 and the Nebraska Center for Materials and Nanoscience.

\section{References}

[1] S. Mangin, D. Ravelosona, J. A. Katine, M. J. Carey, B. D. Terris, and E. E. Fullerton, Nat. Mater. 5 (2006), p. 210.

[2] S. C. Byeon, A. Misra, and W. D. Doyle, IEEE Trans. Magn. 40 (2004), p. 2386.

[3] E. E. Fullerton, D. T. Margulies, Me. E. Schabes, M. Carey, B. Gurney, A. Moser, M. Best, G. Zeltzer, K. Rubin, H. Rosen, and M. Doerner, Appl. Phys. Lett. 77 (2000), p. 3806.

[4] E. N. Abarra, A. Inomata, H. Sato, I. Okamoto, and Y. Mizoshita, Appl. Phys. Lett. 77 (2001), p. 2581.

[5] N. Nishimura, T. Hirai, A. Koganei, T. Ikeda, K. Okano, Y. Sekiguchi, and Y. Osada, J. Appl. Phys. 91 (2002), p. 5246.

[6] P. Grunberg, R. Schreiber, Y. Pang, M. B. Brodsky, and H. Sowers, Phys. Rev. Lett. 57 (1986), p. 2442.

[7] S. S. P. Parkin, N. More, and P. Roche, Phys. Rev. Lett. 64 (1990), p. 2304.

[8] M. D. Stiles, Phys. Rev. B 48 (1993), p. 7238.

[9] P. Bloemen, H. Van Kesteren, H. Swagten, and W. deJonge, Phys. Rev. B 50 (1994), p. 13505.

[10] F. Y. Yang and C. L. Chien, Phys. Rev. Lett. 85 (2000), p. 2597.

[11] F. Ernult, B. Dieny, and J. R. Regnard, J. Magn. Magn. Mater. 242-245 (2002), p. 515.

[12] Yingguo Peng, Jian-Gang Zhu, and David E. Laughlin, J. Appl. Phys. 91 (2002), p. 7676.

[13] B. Heinrich, Y. Tsekovnyak, G. Woltersdorf, A. Brataas, R. Urban, and G. E. W. Bayer, Phys. Rev. Lett. 90 (2003), p. 187601.
[14] J. Camarero, Y. Pennec, J. Vogel, M. Bonfim, and S. Pizzini, Phys. Rev. Lett. 91 (2003), p. 027201.

[15] Z. Y. Liu, Lanping Yue, D. J. Keavney, and S. Adenwalla, Phys. Rev. B 70 (2004), p. 224423.

[16] G. Gubbiotti, S. Tacchi, G. Carlotti, G. Socino, F. Spizzo, Z. Zhao, P. Mani, and G. J. Mankey, J. Magn. Magn. Mater. 286 (2005), p. 468.

[17] A. Baruth, D. J. Keavney, J. D. Burton, K. Janicka, E. Y. Tsymbal, L. Yuan, S. H. Liou, and S. Adenwalla, Phys. Rev. B 74 (2006), p. 054419.

[18] Z. Zhao, C. Papusoi, P. Mani, A. Misra, P. B. Visscher, H. Fujiwara, G. Gubbiotti, S. Tacchi, and G. J. Mankey, IEEE Trans. Magn. 43 (2007), p. 4056.

[19] L. Gao, L. P. Yue, T. Yokota, R. Skomski, S. H. Liou, H. Takahoshi, H. Saito, and S. Ishio, IEEE Trans. Magn. 40 (2004), p. 2194.

[20] S. Michalski, J. Zhou, R. Skomski, and R. D. Kirby, J. Appl. Phys. 101 (2007), p. 09D115.

[21] O. Hellwig, A. Berger, and E. Fullerton, Phys. Rev. Lett. 91 (2003), p. 197203.

[22] S. Hamadaa, K. Himib, T. Okunoa, and K. Takanashi, J. Magn. Magn. Mater. 240 (2002), p. 539.

[23] U. Rucker, S. Demokritov, E. Tsymbal, P. Grunbern, and W. Zinn, J. Appl. Phys. 78 (1995), p. 387.

[24] T. Martin, M. Belmeguenai, M. Maier, K. Perzlmaier, and G. Bayreuther, J. Appl. Phys. 101 (2007), p. 09C101.

[25] M. Desai, A. Misra, and W. D. Doyle, IEEE Trans. Magn. 41 (2005), p. 3151.

[26] A. Moser, A. Berger, D. T. Marhulies, and E. E. Fullerton, J. Appl. Phys. 95 (2004), p. 6657. 